\title{
Diagnosis and Management of Hip Dislocation in Patients with Kabuki Syndrome
}

\author{
Chaemoon Lim, MD, Sung-Taek Jung, MD*, Chang Ho Shin, MD, Moon Seok Park, MD ${ }^{\dagger}$, \\ Won Joon Yoo, MD, Chin Youb Chung, $\mathrm{MD}^{\dagger}$, In Ho Choi, MD, Jung Min Ko, MD ${ }^{\ddagger}$, Tae-Joon Cho, MD \\ Division of Pediatric Orthopaedics, Seoul National University Children's Hospital, Seoul, \\ ${ }^{*}$ Department of Orthopedic Surgery, Chonnam National University Hospital, Gwangju, \\ ${ }^{\dagger}$ Department of Orthopedic Surgery, Seoul National University Bundang Hospital, Seongnam, \\ ${ }^{\ddagger}$ Department of Pediatrics, Seoul National University Children's Hospital, Seoul, Korea
}

Background: Kabuki syndrome is a rare genetic disorder characterized by distinct dysmorphic facial features, growth deficiency, intellectual disabilities, unusual dermatoglyphic patterns, and skeletal abnormalities. The incidence of hip dislocation in Kabuki syndrome ranges from $18 \%$ to $62 \%$. We reviewed the outcomes of management of hip dislocations in patients with Kabuki syndrome with special attention to the diagnostic processes for hip dislocation and Kabuki syndrome.

Methods: Among 30 patients with mutation-confirmed Kabuki syndrome, we selected six patients who had hip dislocations and reviewed their medical records and plain radiographs. The modes of presentation and diagnostic processes for both hip dislocations and Kabuki syndrome were investigated. The management and treatment outcomes of hip dislocations in patients with Kabuki syndrome were evaluated.

Results: The average age of patients at the time of diagnosis of hip dislocation was 7.7 months (range, 1 week to 22 months). None of the patients were diagnosed as having Kabuki syndrome at that time. Two patients were treated with a Pavlik harness; one, with closed reduction; two, with open reduction and later pelvic and/or femoral osteotomies; and one, with open reduction combined with pelvic osteotomy. The patients were followed up for 5.8 years on average (range, 2.0 to 10.5 years). The radiologic outcome was graded as Severin IA or IB for three patients who were older than 6 years at the latest follow-up (mean age, 9.9 years; range, 7.8 to 12.4 years). In the remaining three patients younger than 6 years (mean age, 3.8 years; range, 2.7 to 5.3 years), the lateral center edge angle was more than $15^{\circ}$. The clinical diagnosis of Kabuki syndrome was made during follow-up after hip dislocation treatment and confirmed by mutational analysis at a mean age of 4.7 years. The mean interval between the diagnosis of hip dislocation and Kabuki syndrome was 4.0 years.

Conclusions: The management of hip dislocation by conservative or surgical method showed successful results. Awareness of Kabuki syndrome could lead to an early diagnosis of this rare disease in patients with hip dislocation and allow for early detection of other underlying conditions and multidisciplinary management.

Keywords: Kabuki syndrome, Hip dislocation

Received April 16, 2019; Accepted July 26, 2019

Correspondence to: Tae-Joon Cho, MD

Division of Pediatric Orthopaedics, Seoul National University Children's

Hospital, 101 Daehak-ro, Jongno-gu, Seoul 03080, Korea

Tel: +82-2-2072-2878, Fax: +82-2-2745-3367

E-mail: tjcho@snu.ac.kr
Kabuki syndrome is a rare genetic disorder with distinct dysmorphic facial features, postnatal growth deficiency, intellectual disabilities, unusual dermatoglyphic patterns, and skeletal abnormalities. ${ }^{1)}$ Kabuki syndrome was first described in Japan in $1981,{ }^{1,2)}$ and thereafter has been reported in a variety of ethnic groups. ${ }^{3-5)}$ Heterozygous mutations in the KMT2D or KMT6D genes were reported 
to cause Kabuki syndrome, with $55.8 \%$ to $80 \%$ of patients having the former gene mutation ${ }^{6-8)}$ and $12.5 \%$ having the latter mutation. ${ }^{9)}$ These mutations involve abnormal chromatin remodeling and transcriptional dysregulation. ${ }^{10)}$ However, in $30 \%$ of the patients, no mutation was identified in either of the two genes, suggesting the presence of another causative gene for this phenotype. ${ }^{10)}$

The dysmorphic facial features of patients with Kabuki syndrome include a long palpebral fissure, eversion of the lateral one-third of the lower eyelid, an arched eyebrow with sparseness in the lateral one-third, short columella with a depressed nasal tip, and prominent ears. ${ }^{1)}$ All these features bear resemblance to the actors' makeup in Kabuki, the traditional Japanese theatre. ${ }^{1)}$ Patients with Kabuki syndrome may have anomalies or dysfunction of the cardiac, urogenital, otolaryngological, ophthalmological, endocrine, and skeletal systems, along with ligamentous hyperlaxity. ${ }^{1)}$ The skeletal abnormalities consist of scoliosis and vertebral malformations, ${ }^{1,2)}$ clavicle pseudoarthrosis, ${ }^{11)}$ brachydactyly and clinodactyly of the fifth finger, ${ }^{1,2)}$ hip and/or patellar dislocation, ${ }^{1,2,12,13)}$ tarsal coalition, and clubfeet. $\left.{ }^{14}\right)$ The incidence of hip dislocations in Kabuki syndrome ranges from $18 \%$ to $62 \%$. ${ }^{5,15)}$ Some studies showed that most of the hip dislocations in Kabuki syndrome could be treated successfully with surgical interventions. ${ }^{15,16)}$ In this study, we investigated the diagnostic process for Kabuki syndrome and hip dislocation and the outcomes of treatment of hip dislocation in patients with Kabuki syndrome.

\section{METHODS}

This retrospective study was reviewed and approved by the Institutional Review Board of Seoul National University Hospital (IRB No. 1805-139-948). We conducted this study in compliance with the principles of the Declaration of Helsinki. The informed consent was waived. We reviewed the medical records and radiographs of Kabuki syndrome patients. Between 2002 and 2018, 30 patients were diagnosed as having Kabuki syndrome at our institution. Kabuki syndrome was diagnosed clinically based on the recognition of its five main characteristics (distinct dysmorphic facial features, postnatal growth deficiency, intellectual disabilities, unusual dermatoglyphic patterns, and skeletal abnormalities) and was confirmed by KMT2D or KDM6A mutational studies.

Among 30 mutation-confirmed Kabuki syndrome patients, six patients were diagnosed as having hip dislocations. The hip dislocations were confirmed with ultrasonography or by Tönnis grade on plain radiographs. Management methods for the hip dislocations were reviewed from medical records. The outcomes of management of hip dislocation were assessed by lateral center edge angle (CEA) and Severin classification in plain radiographs of the hips. We reviewed the modes of presentation and diagnostic process for both hip dislocation and Kabuki syndrome. We also analyzed how Kabuki syndrome were recognized in hip dislocation patients and reviewed the interval between the diagnosis of hip dislocation and Kabuki syndrome.

\section{Table 1. Management and Results of Hip Dislocation}

\begin{tabular}{|c|c|c|c|c|c|c|c|}
\hline Case & Sex & Side & Status of hip & Treatment & $\begin{array}{l}\text { Lateral CEA at } \\
\text { last follow-up }\left(^{\circ}\right)\end{array}$ & $\begin{array}{l}\text { Age at the last } \\
\text { follow-up (yr) }\end{array}$ & $\begin{array}{l}\text { Follow-up } \\
\text { period (yr) }\end{array}$ \\
\hline \multirow[t]{2}{*}{1} & $\mathrm{~F}$ & Left & Graf type IV & Pavlik harness & & 2.7 & 2.7 \\
\hline & & Right & Graf type D & Pavlik harness & & & \\
\hline \multirow[t]{2}{*}{2} & $F$ & Left & Graf type IV & Pavlik harness & 20 & 9.4 & 9.2 \\
\hline & & Right & Graf type Ila (-) & Pavlik harness & 19 & & \\
\hline 3 & $F$ & Left & Dislocation & $\mathrm{CR}$ & 20 & 12.4 & 10.5 \\
\hline 4 & $\mathrm{~F}$ & Left & Dislocation & $\mathrm{OR} \rightarrow \mathrm{FVO}$ & 16 & 7.8 & 6.0 \\
\hline 5 & M & Left & Dislocation & $\mathrm{OR} \rightarrow \mathrm{FVDO} \& \mathrm{PO}$ & 18 & 5.3 & 4.5 \\
\hline 6 & $\mathrm{~F}$ & Left & Dislocation & OR \& PO & 20 & 3.3 & 2.0 \\
\hline Mean & & & & & & & 5.8 \\
\hline
\end{tabular}

CEA: center edge angle, F: female, M: male, CR: closed reduction, OR: open reduction, FVO: femoral varization osteotomy, FVDO: femoral varization derotational osteotomy, $\mathrm{PO}$ : pelvic osteotomy. 


\section{RESULTS}

There were five females and one male included in this study. The mean age at hip dislocation diagnosis was 7.7 months (range, 1 week to 22 months). Kabuki syndrome was not recognized in all patients at that time. The mean follow-up period for hip dislocation was 5.9 years (range, 2.7 to 10.5 years). The mean age at the last follow-up was 9.9 years (range, 2.7 to 12.4 years).

Two female patients were referred to the orthopedic clinic because of abnormal physical examinations during neonatal hip screening. One patient showed positive Ortolani and Barlow test, positive Galeazzi sign, and asymmetric inguinal skin fold. The other patient was referred from another hospital at 2 months old. We could not obtain the exact neonatal hip screening record for this patient. Hip dislocation was diagnosed by ultrasonography in both patients. One patient was found to have a Graf type IV hip on the left side and a Graf type D hip on the right side at 1 week of age. She was treated with a Pavlik harness for 2 months. Both hip joints remained concentrically reduced at age 2.7 years. The other patient was found to have a Graf type IV hip on the left side and a Graf type IIa (-) on the right side at 7 weeks of age. She was also treated with a Pavlik harness for 2 months. At the last follow-up, at age 9.4 years, the lateral CEA was $19^{\circ}$ on the right hip and $20^{\circ}$ on the left hip, and the Severin class was IA (Table 1 and Fig. $1)$.

Four patients were referred for asymmetry of the ischiogluteal skin fold, limitation of hip abduction, or limping gait at ages of 1.8 years, 1 year, 9 months, and 5 months. In them, hip dislocation was diagnosed by plain radiographs and Tönnis grades were 2, 3, 2, and 2. One of them was treated by closed reduction at age 1.8 years. At
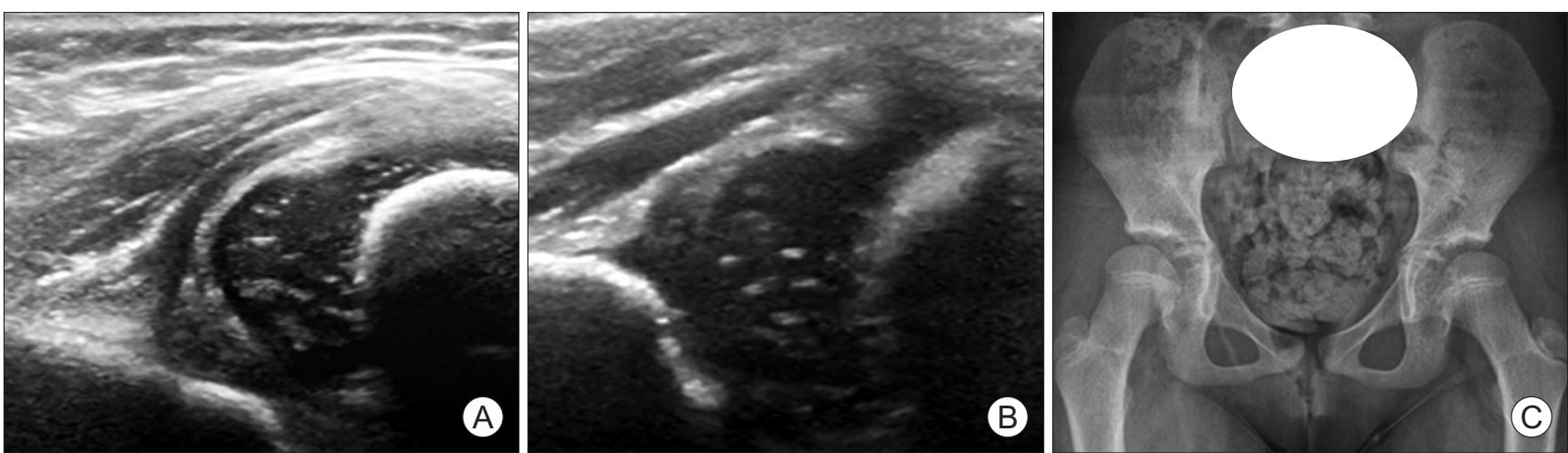

Fig. 1. Case 2, treated with a Pavlik harness. Ultrasonographic images of a 7-week-old female patient showing left hip dislocation of Graf type IV (A) and right hip dysplasia of Graf type lla (-) (B). (C) Radiograph at 9.4 years of age: lateral center edge angle was $20^{\circ}$ and Severin class was IA.
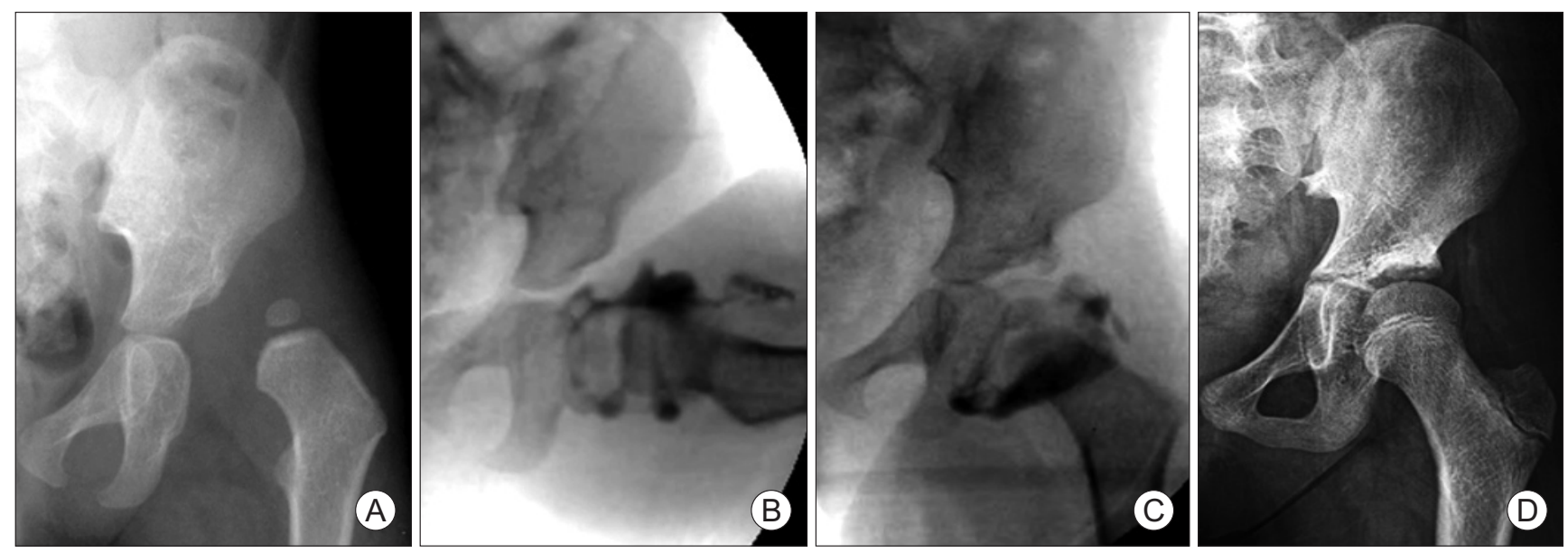

Fig. 2. Case 3, treated by closed reduction. (A) Initial radiograph of a 1.8-year-old female patient showing left hip dislocation of Tönnis grade 3. (B) Intraoperative arthrogram after closed reduction, showing concentric reduction of the left hip joint. (C) Concentric reduction was maintained at 2 months after closed reduction. (D) Radiograph at 12.4 years of age: lateral center edge angle was $20^{\circ}$ and Severin class was IA. 
age 12.4 years, the lateral CEA was $20^{\circ}$, and the hip was classified as Severin IA (Table 1 and Fig. 2).

Two of the patients were initially treated by anterolateral open reduction at age 1 year and by medial open reduction at age 9 months. These two patients developed residual dysplasia or subluxation during follow-up and underwent femoral or pelvic osteotomy or both at age 3.6 and 2.8 years. In one of them, the hip was classified as Severin IB with a CEA of $16^{\circ}$ at age 7.8 years (Table 1 and Fig. 3 ); in the other, the CEA was $18^{\circ}$ at age 5.3 years.

The remaining one female patient was treated by open reduction and simultaneous pelvic osteotomy at age 21 months. In her, left hip dislocation was diagnosed at age
5 months but treatment was delayed because of surgery for a cleft palate. At age 3.3 years, the hip joint remained concentrically reduced with a lateral CEA of $20^{\circ}$.

In all patients, hip dislocation was diagnosed before the diagnosis of Kabuki syndrome. Although other systemic manifestations and facial dysmorphism were individually identified, Kabuki syndrome was not suspected for a certain period of time. Two patients had cardiac malformation: one patient with a ventricular septal defect underwent spontaneous closure; the other, with hypoplastic left heart syndrome, had open heart surgery before hip dislocation treatment. Three patients had cleft palates, and two of them had palatoplasty. Four patients had urogenital
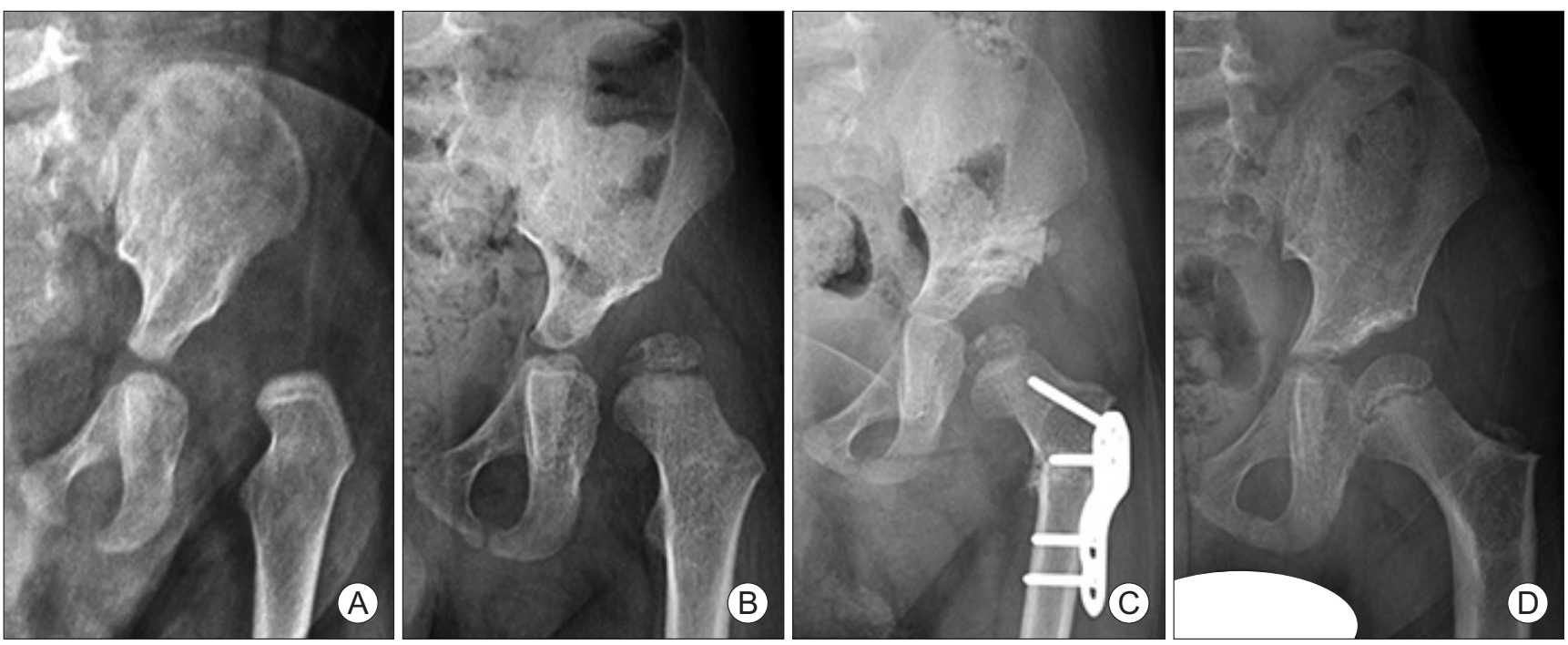

Fig. 3. Case 5, treated by open reduction and osteotomy. (A) Initial radiograph of a 9-month-old male patient, showing left hip dislocation of Tönnis grade 2. (B) At age 2.8 years, the left hip remained subluxated laterally with acetabular dysplasia after medial approach open reduction performed at 9 months. (C) Femoral varization osteotomy and Dega osteotomy were performed. (D) Radiograph at 5.3 years of age: the left hip remained in concentric reduction state with a center edge angle of $18^{\circ}$.

Table 2. Systemic Manifestations

\begin{tabular}{|c|c|c|c|c|c|}
\hline Case & Cardiac malformation & Cleft palate & Urogenital abnormality & $\begin{array}{l}\text { Otolaryngological } \\
\text { abnormality }\end{array}$ & Mental retardation \\
\hline 1 & Ventricular septal defect & Yes & Ectopic kidney & & \\
\hline 2 & & Yes (palatoplasty) & Single kidney & Middle ear effusion & Yes \\
\hline 3 & & & & & Yes \\
\hline 4 & $\begin{array}{l}\text { Hypoplastic left heart } \\
\text { syndrome (open heart } \\
\text { surgery) }\end{array}$ & & Multicystic kidney disease & $\begin{array}{l}\text { Middle ear effusion } \\
\text { (Ventilation tube insertion) }\end{array}$ & Yes \\
\hline 5 & & & & & Yes \\
\hline 6 & & Yes (palatoplasty) & Horsehoe kidney & $\begin{array}{l}\text { Middle ear effusion } \\
\text { (Ventilation tube insertion) }\end{array}$ & Yes \\
\hline
\end{tabular}


Lim et al. Hip Dislocation in Kabuki Syndrome

Clinics in Orthopedic Surgery • Vol. 11, No. 4, $2019 \bullet$ www.ecios.org

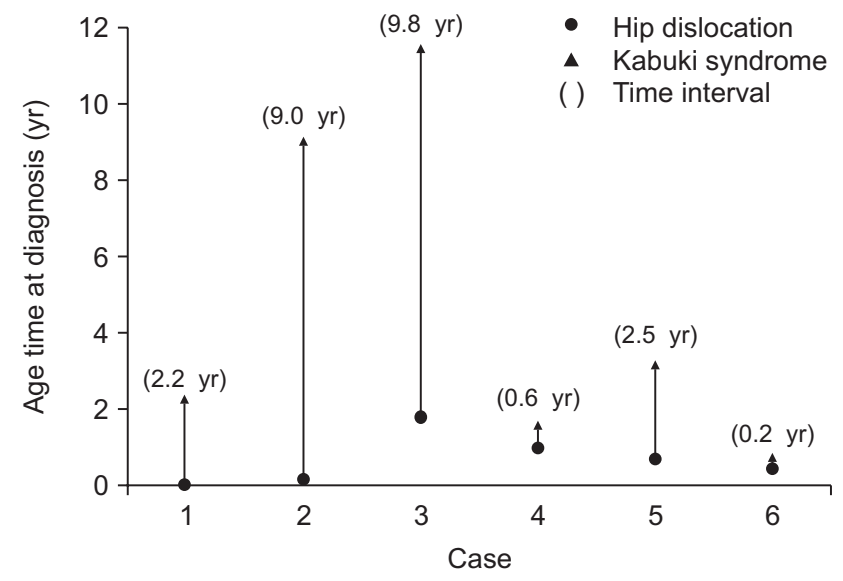

Fig. 4. Age at the time of diagnosis of hip dislocation and Kabuki syndrome. The mean age at hip dislocation diagnosis was 7.7 months (range, 1 week to 22 months). The mean age at the time of Kabuki syndrome diagnosis was 4.7 years (range, 0.6 to 11.6 years). The mean interval from the diagnosis of hip dislocation to the diagnosis of Kabuki syndrome was 4.0 years (range, 0.2 to 9.8 years).

abnormalities. Three patients had otolaryngological abnormality (middle ear effusion) and two patients among them had ventilation tube insertion surgery. Five patients were mentally retarded (Table 2). As the facial dysmorphism became more obvious, surgeons started to suspect Kabuki syndrome. The patients were referred to a clinical geneticist and were diagnosed as having Kabuki syndrome. All six patients were found to harbor mutations in the KMT2D gene. The mean age at the time of diagnosis of Kabuki syndrome was 4.7 years (range, 0.6 to 11.6 years). The mean interval from the diagnosis of hip dislocation to the diagnosis of Kabuki syndrome was 4.0 years (range, 0.2 to 9.8 years) (Fig. 4 ).

\section{DISCUSSION}

We reported treatment outcomes of hip dislocation associated with Kabuki syndrome in six patients and reviewed how the diagnoses of hip dislocation and Kabuki syndrome were made. There are two previous reports of treatment of hip dislocation in Kabuki syndrome patients. Ramachandran et al. ${ }^{15)}$ reported two Kabuki syndrome children with three hip dislocations. At around walking age, the patients underwent open reduction combined with Dega osteotomy. The hip dislocations in Kabuki syndrome were managed in a similar manner to neglected developmental dysplasia of the hip and the results were satisfactory. Wada et al. ${ }^{16)}$ also reported the treatment outcomes of five Kabuki syndrome children with seven hip dislocations. Five hip dislocations were diagnosed before
10 months of age, and nonoperative treatment (closed reduction) resulted in successful reduction and maintenance. However, after walking age, subluxation or redislocation occurred in these five hips. Hip dislocation in the other two patients was diagnosed at 2.4 years of age. Hence, they performed open reduction along with femoral and pelvic osteotomy at an average age of 3.6 years (range, 2.4 to 5.7 years) in all seven patients with hip dislocation. They reported that acetabular deficiency in Kabuki syndrome was more posterior than that in idiopathic developmental dysplasia of the hip and similar to that in neuromuscular disorders and Down syndrome. To provide sufficient posterolateral coverage, they performed an incomplete periacetabular osteotomy alternative to Salter or conventional Dega osteotomy. It was similar to the modified Dega osteotomy technique used in cerebral palsy patients, in which a wedge bone graft was inserted posteriorly to ensure posterosuperior coverage. ${ }^{17-19)}$

In our study, three hips were successfully treated with nonoperative methods (Pavlik harness or closed reduction or both). They remained concentrically reduced and developed normally for 2.7 to 10 years of follow-up. Although a longer follow-up period is needed to confirm normal development of the hip joint, deep concentric reduction was confirmed at the latest follow-up. These results show that Pavlik harness or closed reduction, which is used to manage the idiopathic developmental dysplasia of the hip, can be a useful treatment tool for hip dislocation in the infant period of Kabuki syndrome. In the remaining three hips, residual dysplasia or subluxation developed, which were addressed by femoral or pelvic osteotomy or both. Although we currently use threedimensional computed tomography (3D-CT) to evaluate acetabular morphology in candidates of acetabular osteotomy, it was not used routinely at that time. None of the six patients underwent 3D-CT. We did not find whether there was posterior acetabular deficiency in our cases undergoing pelvic osteotomies. But the acetabular dysplasia was successfully treated with the conventional Dega osteotomy technique.

The major pathology of hip dislocation in Kabuki syndrome is ligamentous laxity ${ }^{16)}$ as in Down syndrome, Ehlers-Danlos syndrome, and Larsen syndrome. According to the literature, hip dislocations of Down syndrome are associated with ligamentous laxity, excessive femoral anteversion, and posterior acetabular wall deficiency. ${ }^{20}$ According to Aly and Al-Kersh, ${ }^{20)}$ osteotomy is always required in hip dislocation in Down syndrome. They combined femoral varization-derotation osteotomies and acetabular derotation osteotomies for posterior acetabular 
coverage. Ehlers-Danlos syndrome is another example of hip dislocations resulting from ligamentous laxity. Hip dislocations in Ehlers-Danlos syndrome have been reported in $1 \%$ to $4 \%$ of the patients. ${ }^{21)}$ Badelon et al. ${ }^{22)}$ and Giunta et al. ${ }^{23)}$ suggested that hip dislocation treatment in Ehlers-Danlos syndrome patients should consist of open reduction with both pelvic and femoral osteotomies. They suggested that hip dislocation associated with ligamentous laxity requires more bony stability of the hip joint. Wada et al. ${ }^{16)}$ reported that the acetabular deficiency in Kabuki syndrome patients was more posterior and performed an incomplete periacetabular osteotomy to provide sufficient posterolateral coverage. We agree that if the hip joint remains unstable or dysplastic, bony stabilization is indicated. But our results suggest that the acetabular dysplasia in Kabuki syndrome could be addressed with the conventional Dega osteotomy technique as in the treatment of developmental dysplasia of the hip.

Ramachandran et al. ${ }^{15)}$ reported that the neonatal hip screening tests carried out by non-orthopedists did not detect hip dysplasia in their two patients. This may have been due to either late-onset hip instability or the failure to detect hip dysplasia in neonatal hip screening tests. They emphasized that orthopedic surgeons should be aware of difficulties diagnosing hip dislocations in patients with Kabuki syndrome, especially in patients with bilateral hip dislocation and ligamentous laxity. We agree that the orthopedist should consider the absence of abnormal physical findings when examining hip dislocation patients with ligamentous laxity, not just those with Kabuki syndrome. In our study, the two patients in whom hip dislocation was diagnosed early via abnormal physical examinations during neonatal hip screening were successfully treated with Pavlik harness. This suggests that the early diagnosis of hip dislocation via neonatal hip screening is important for nonoperative treatment of Kabuki syndrome.

The clinical diagnosis of Kabuki syndrome is challenging during the neonatal period because the characteristic facial features become evident only in childhood. ${ }^{4)}$ Vaux et al. ${ }^{24)}$ reviewed 16 cases of Kabuki syndrome. The average age of the initial evaluation by a dysmorphologist was 8 days after birth (range, birth to 28 days) but the clinical diagnosis of Kabuki syndrome was delayed, made at an average age of 2.5 years (range, 2 days to 8.5 years). Although the diagnosis of Kabuki syndrome was not made during the neonatal period, many of the typical facial features were present at that time. The authors ${ }^{24)}$ suggested that the diagnosis or at least consideration of Kabuki syndrome in neonates is possible.

No study has examined the interval between the di- agnosis of hip dislocation and that of Kabuki syndrome. In previous studies of hip dislocations in Kabuki syndrome patients, the hip dislocations were described in known Kabuki syndrome patients. There was no information regarding diagnostic timing of the hip dislocations versus Kabuki syndrome. However, in our series, hip dislocations were diagnosed earlier than Kabuki syndrome in all cases. Two patients were diagnosed with hip dislocations by neonatal hip screening and were referred to us. The remaining four patients presented to our clinic with symptoms of hip dislocation after 5 months old. The diagnosis of hip dislocation was made at the first visit to our clinic at an average of 7.7 months of age. Although they had dysmorphic facial feature and/or other clinical manifestations including cardiac, urogenital, and otolaryngological anomalies, at the first presentation, the orthopedists who first examined the patients failed to correlate those manifestations with Kabuki syndrome. Kabuki syndrome was suspected sometime during the management of hip dislocations, and the patients were referred to a pediatric geneticist, who made the clinical and molecular diagnosis of Kabuki syndrome. As a result, the diagnosis of Kabuki syndrome was delayed by a mean of 2.8 years.

Although the clinical diagnosis of Kabuki syndrome in the neonatal period is difficult, a delayed diagnosis of Kabuki syndrome may cause the associated medical problems to go unnoticed or the patients to undergo unnecessary diagnostic workup. On the contrary, an early diagnosis of Kabuki syndrome may lead to the prospective care of associated medical conditions and genetic counselling. ${ }^{25)}$ Hip dislocation may be one of the early presenting manifestations of Kabuki syndrome that require medical attention in the neonatal period. Hence, it would be worthwhile for orthopedists involved in hip dislocation management to be aware of this rare disease.

There are some limitations inherent in this study. Because it was a retrospective case series, the age at the last follow-up and the follow-up period were variable, which might have caused a bias in assessing the effect of hip dislocation management. All of the patients did not reach skeletal maturity and the sample size was small. In addition, we did not investigate whether there was posterior acetabular deficiency. However, the cases treated with conventional Dega osteotomy showed good results at the last follow-up. Therefore, we think the conventional Dega osteotomy is a viable option for management of pelvic dysplasia in Kabuki syndrome.

Kabuki syndrome is frequently associated with hip dislocation probably due to ligamentous laxity and could be successfully managed by conservative treatment if de- 
tected early. Residual dysplasia or subluxation requires bony procedures to stabilize the hip joint. Because hip dislocation is one of the early manifestations of Kabuki syndrome, awareness of this rare disease by pediatric orthopedic surgeons is crucial for early diagnosis of Kabuki syndrome and proper diagnosis and management of other systemic problems.

\section{CONFLICT OF INTEREST}

No potential conflict of interest relevant to this article was reported.

\section{ACKNOWLEDGEMENTS}

This research was supported by Genome Technology to Business Translation Program of the National Research Foundation (NRF) funded by the Ministry of Science, ICT \& Future Planning (NRF-2014M3C9A2064684).

\section{REFERENCES}

1. Niikawa N, Matsuura N, Fukushima Y, Ohsawa T, Kajii T. Kabuki make-up syndrome: a syndrome of mental retardation, unusual facies, large and protruding ears, and postnatal growth deficiency. J Pediatr. 1981;99(4):565-9.

2. Kuroki Y, Suzuki Y, Chyo H, Hata A, Matsui I. A new malformation syndrome of long palpebral fissures, large ears, depressed nasal tip, and skeletal anomalies associated with postnatal dwarfism and mental retardation. J Pediatr. 1981;99(4):570-3.

3. Niikawa N, Kuroki Y, Kajii T, et al. Kabuki make-up (Niikawa-Kuroki) syndrome: a study of 62 patients. Am J Med Genet. 1988;31(3):565-89.

4. Philip N, Meinecke P, David A, et al. Kabuki make-up (Niikawa-Kuroki) syndrome: a study of 16 non-Japanese cases. Clin Dysmorphol. 1992;1(2):63-77.

5. Schrander-Stumpel C, Meinecke P, Wilson G, et al. The Kabuki (Niikawa-Kuroki) syndrome: further delineation of the phenotype in 29 non-Japanese patients. Eur J Pediatr. 1994;153(6):438-45.

6. Banka S, Veeramachaneni R, Reardon W, et al. How genetically heterogeneous is Kabuki syndrome?: MLL2 testing in 116 patients, review and analyses of mutation and phenotypic spectrum. Eur J Hum Genet. 2012;20(4):381-8.

7. Li Y, Bogershausen $\mathrm{N}$, Alanay $\mathrm{Y}$, et al. A mutation screen in patients with Kabuki syndrome. Hum Genet. 2011;130(6):71524.

8. Ng SB, Bigham AW, Buckingham KJ, et al. Exome sequencing identifies MLL2 mutations as a cause of Kabuki syndrome. Nat Genet. 2010;42(9):790-3.

9. Bogershausen N, Wollnik B. Unmasking Kabuki syndrome. Clin Genet. 2013;83(3):201-11.

10. Lintas C, Persico AM. Unraveling molecular pathways shared by Kabuki and Kabuki-like syndromes. Clin Genet.
2018;94(3-4):283-95.

11. Hinrichs B, Gramss B, Meinecke P. Defective clavicles in Kabuki syndrome. Genet Couns. 2002;13(4):477-9.

12. Ikegawa S, Sakaguchi R, Kimizuka M, Yanagisako Y, Tokimura F. Recurrent dislocation of the patella in Kabuki make-up syndrome. J Pediatr Orthop. 1993;13(2):265-7.

13. Kurosawa K, Kawame H, Ochiai Y, Nakashima M, Tohma T, Ohashi H. Patellar dislocation in Kabuki syndrome. Am J Med Genet. 2002;108(2):160-3.

14. Phillips S, Hemmady S, Thomas P, ODoherty D. Kabuki syndrome presenting with congenital talipes equinovarus. J Pediatr Orthop B. 2005;14(4):285-6.

15. Ramachandran M, Kay RM, Skaggs DL. Treatment of hip dislocation in Kabuki syndrome: a report of three hips in two patients. J Pediatr Orthop. 2007;27(1):37-40.

16. Wada A, Nakamura T, Yamaguchi T, et al. Surgical treatment of hip dislocation in Kabuki syndrome: use of incomplete periacetabular osteotomy for posterior acetabular wall deficiency. J Child Orthop. 2012;6(4):261-7.

17. Chung CY, Park MS, Choi IH, Cho TJ, Yoo WJ, Lee KM. Morphometric analysis of acetabular dysplasia in cerebral palsy. J Bone Joint Surg Br. 2006;88(2):243-7.

18. Chung CY, Choi IH, Cho TJ, Yoo WJ, Lee SH, Park MS. Morphometric changes in the acetabulum after Dega osteotomy in patients with cerebral palsy. J Bone Joint Surg Br. 2008;90(1):88-91.

19. Kim HT, Jang JH, Ahn JM, Lee JS, Kang DJ. Early results of one-stage correction for hip instability in cerebral palsy. Clin Orthop Surg. 2012;4(2):139-48.

20. Aly AS, Al-Kersh MA. Femoral and Dega osteotomies in the treatment of habitual hip dislocation in Down syndrome patients: is it efficient or not? J Child Orthop. 2018;12(3):227- 
Lim et al. Hip Dislocation in Kabuki Syndrome

Clinics in Orthopedic Surgery • Vol. 11, No. 4, $2019 \bullet$ www.ecios.org

31.

21. Beighton P, Horan F. Orthopaedic aspects of the EhlersDanlos syndrome. J Bone Joint Surg Br. 1969;51(3):444-53.

22. Badelon O, Bensahel H, Csukonyi Z, Chaumien JP. Congenital dislocation of the hip in Ehlers-Danlos syndrome. Clin Orthop Relat Res. 1990;(255):138-43.

23. Giunta C, Superti-Furga A, Spranger S, Cole WG, Stein- mann B. Ehlers-Danlos syndrome type VII: clinical features and molecular defects. J Bone Joint Surg Am. 1999;81(2):225-38.

24. Vaux KK, Hudgins L, Bird LM, et al. Neonatal phenotype in Kabuki syndrome. Am J Med Genet A. 2005;132(3):244-7.

25. Dentici ML, Di Pede A, Lepri FR, et al. Kabuki syndrome: clinical and molecular diagnosis in the first year of life. Arch Dis Child. 2015;100(2):158-64. 